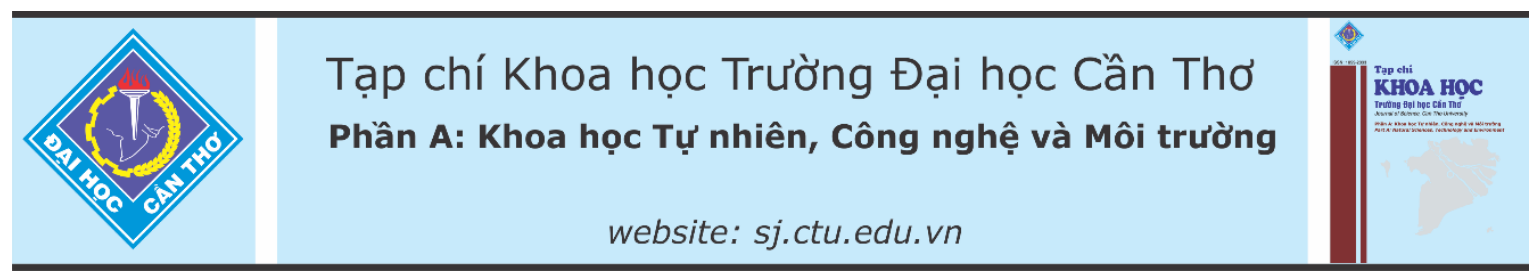

DOI:10.22144/ctu.jvn.2021.011

\title{
XÂY DỬNG KHÔNG GIAN TÔPÔ MỀM TRUNG TÍNH TRÊN CÁC PHÉP TOÁN MỚI
}

Trần Thị Bảo Trâm*

Khoa Khoa học Đại cuoong, Truờng Đại học Tài Nguyên và Môi Truờng TP. Hồ Chí Minh

*Người chịu trách nhiệm về bài viết: Trần ThịBảoTrâm (email: ttbtram@hcmunre.edu.vn)

\section{Thông tin chung:}

Ngày nhận bài: $26 / 08 / 2020$

Ngày nhận bài sưa: 29/09/2020

Ngày duyệt đăng: 27/03/2021

Title:

Constructing neutrosophic soft topological space on new operations

\section{Tù̀ khóa:}

Tập mềm trung tính, không gian tôpô mềm trung tính

\section{Keywords:}

Neutrosophic soft set, neutrosophic soft topological space

\begin{abstract}
The main goal of this paper is to construct the neutrosophic soft topogical space by giving new operations on neutrosophic soft set. The author has changed the operations constructed in the research of Ozturk and et al. (2019) based on original definitions: fuzzy sets, soft sets, neutrosophic soft sets. Then, we have checked the properties and propositions of these operations. Finally, we want to clarify new constructed operations keep the relationship of neutrosophic soft topological space and component topogical spaces: fuzzy topogical space, fuzzy soft topogical space.
\end{abstract}

\section{TÓM TẮT}

Mục tiêu chính của bài viết này là xây dưng không gian tôpô trên tập mềm trung tính bằng cách đura ra các phép toán mới. Bài viết thay thế các phép toán đã đưa ra trong nghiên cứu của Ozturk et al. (2019). bằng các phép toán mới trên nền các định nghĩa gốc về tập mờ tập mềm, tập mềm trung tính. Tù đó, chúng tôi kiểm tra lại các tính chất và định lý của các phép toán này. Cuối cùng, bài viết cũng muốn chứng tỏ các phép toán này đảm bảo việc giữ mối liên hệ giũa không gian tôpô mềm trung tính và các không gian tôpô thành phần: không gian tôpô mò̀, không gian tôpô mềm $m o$.

\section{GIỚI THIỆ}

Nhiều năm gần đây, các công cụ toán học hỗ trợ rất nhiều cho lĩnh vực khai thác thông tin trên dữ liệu. Các nghiên cứu được thực hiện bởi rất nhiều nhà toán học đã giải quyết được các vấn đề phát sinh. Trong các vấn đề ở các lĩnh vực như môi trường, kinh tế, khoa học xã hội, khoa học y tế, quản trị kinh doanh và kỹ thuật, khi các nhà nghiên cứu sử dụng các mô hình toán cổ điển để thu thập thông tin, dữ liệu không chắc chắn làm tăng thêm tính phức tạp và khó khăn trong quá trình thực hiện. Để tránh những điều này, nhiều công cụ toán mới, mạnh hơn được nghiên cứu để giải quyết những khó khăn trên. Một trong số các công cụ đó là tập mờ. Nhưng nhược điểm của chúng là không thể xử lý những thông tin không xác định và không nhất quán. Sau đó, khái niệm tập trung tính được giới thiệu bởi Smarandache trở thành một công cụ toán học giải quyết được các vấn đề liên quan đến dữ liệu không chính xác, không xác định. Sau đó, Molodtsov đã đưa ra lý thuyết tập mềm, lý thuyết này là một cách tiếp cận hoàn toàn mới giúp cho việc mô hình hóa sự không chắc chắn và áp dụng nó một cách thành công trong một số hướng nghiên cứu như hàm trơn, lý thuyết trò chơi, nghiên cứu toán tử,.... Tiếp theo, Maji giới thiệu một khái niệm kết hợp, đó là tập mềm trung tính. Sử dụng khái niệm này, các nhà toán học đã xây dựng nhiều cấu trúc toán học khác nhau trong các nghiên cứu của họ. Trong bài viết này, chúng tôi giới thiệu các phép toán giao, hợp, 
hiệu, AND, OR trên tập mềm trung tính. Các phép toán này hoàn toàn khác so với các phép toán đã đề cập trong bài báo của Ozturk et al. (2019). Từ đó, chúng tôi kiểm tra một số tính chất của các phép toán trên nền lý thuyết tập hợp cổ điển. Thêm vào đó, bài viết cũng xây dựng lại không gian tôpô mềm trung tính, kiểm tra mối liên hệ giữa không gian tôpô mềm trung tính, không gian tôpô mềm mờ và không gian tôpô mờ.

\section{PHƯƠNG PHÁP NGHIÊN CÚU}

Ozturk et al. (2019) đã đưa ra các phép toán giao, hợp, hiệu, AND, OR trên các tập mềm trung tính. Từ đó, các tác giả đưa ra các tính chất có được từ các phép toán này, xây dựng không gian tôpô mềm trung tính, kiểm tra mối liên hệ giữa các không gian tôpô: không gian tôpô mềm trung tính, không gian tôpô mềm mờ, không gian tôpô mờ. Một câu hỏi đặt ra là liệu khi thay đồi định nghĩa các phép toán trên các tập mềm trung tính thì các tính chất có được bảo toàn? Từ đó việc xây dựng không gian tôpô trên tập mềm trung tính có gì khác? Mối liên hệ giữa các không gian tôpô đã đề cập còn được đảm bảo khi đưa ra các phép toán mới? Bài viết này sẽ đưa ra các phép toán mới và trả lời các câu hỏi trên. Để tiện cho người đọc theo dõi, phần đầu được trình bày theo trình tự sau: phần đầu, bài viết nhắc lại các định nghĩa gốc trong các tài liệu trước đó như tập mềm, tập trung tính và tập mềm trung tính. Tiếp theo, chúng tôi đưa ra các phép toán mới và kiểm tra các tính chất.

\subsection{Các định nghĩa cần thiết}

Định nghĩa 2.1 (Smarandache, 2005) Xét tập vũ trụ $X$. Tập trung tính $A$ trên $X$ được xác định như sau: $A=\left\{\left\langle x, T_{A}(x), I_{A}(x), F_{A}(x)\right\rangle: x \in X\right\}$, trong đó $T, I, F: X \rightarrow[0,1]$ và hiển nhiên $0 \leq T_{A}(x)+$ $I_{A}(x)+F_{A}(x) \leq 3$.

Định nghĩa 2.2 (Molodtsov, 1999) Cho $X$ là tập vũ trụ và $E$ là tập các tham số. Gọi $P(X)$ là tập chứa các tập con của $X$. Khi đó, với mọi $A \subseteq E$, cặp $(G, A)$ được gọi là tập mềm trên $X$, trong đó $G$ là một ánh xạ được mô tả như sau: $G: E \rightarrow P(X)$, thỏa $(G, A)=\{(e, G(a)): e \in A, G: A \rightarrow P(X)\}$.

Định nghĩa 2.3 (Deli and Broumi, 2015) Cho $X$ là tập vũ trụ và $E$ là tập các tham số. Gọi $N(X)$ là tập chứa tất cả các tập trung tính trên $X$. Khi đó $(G, E)$ được gọi là một tập mềm trung tính trên $X$ nếu $G: E \rightarrow N(X)$ là một ánh xạ. Nói một cách khác, $(G, E)$ là tập chứa các bộ được sắp thứ tự có dạng cụ thể như sau: $(G, E)=\left\{\left(e,\left\langle x, T_{G(e)}(x), I_{G(e)}(x)\right.\right.\right.$, $\left.\left.\left.F_{G(e)}(x)\right\rangle: x \in X\right): e \in E\right\}$, trong đó $T_{G(e)}(x)$, $I_{G(e)}(x), F_{G(e)}(x) \in[0,1] \quad$ và $0 \leq T_{G(e)}(x)+$ $I_{G(e)}(x)+F_{G(e)}(x) \leq 3$.

Định nghĩa 2.4 (Bera and Mahapatra, 2017) Xét $\left(G_{1}, E\right)$ và $\left(G_{2}, E\right)$ là hai tập mềm trung tính trên tập vũ trụ $X$. $\left(G_{1}, E\right)$ được gọi là tập con của $\left(G_{2}, E\right)$, ký hiệu $\left(G_{1}, E\right) \subseteq\left(G_{2}, E\right)$ nếu $T_{G_{1}(e)}(x) \leq T_{G_{2}(e)}(x)$; $I_{G_{1}(e)}(x) \leq I_{G_{2}(e)}(x) ; F_{G_{1}(e)}(x) \geq F_{G_{2}(e)}(x), \quad$ với mọi $e \in E, x \in X$.

$\left(G_{1}, E\right)$ bằng $\left(G_{2}, E\right)$ nếu $\left(G_{1}, E\right)$ là tập con mềm trung tính của $\left(G_{2}, E\right)$ và ngược lại, ký hiệu là $\left(G_{1}, E\right)=\left(G_{2}, E\right)$.

Định nghĩa 2.5 (Maji, 2013) Cho $(G, E)$ là tập mềm trung tính trên $X$. Phần bù của $(G, E)$, ký hiệu là $(G, E)^{C}$ được xác định như sau: $(G, E)^{C}=$ $\left\{\left(e,\left\langle x, F_{G(e)}(x), 1-I_{G(e)}(x), T_{G(e)}(x)\right\rangle: x \in\right.\right.$ $X): e \in E\}$. Ta có, $\left((G, E)^{C}\right)^{C}=(G, E)$.

\subsection{Xây dựng các phép toán mới}

Trong phần này, tác giả định nghĩa mới các phép toán, bao gồm: hợp, giao, hiệu,...trên các tập mềm trung tính. Lưu ý rằng, các phép toán này hoàn toàn khác so với các phép toán đã được xây dựng trong nghiên cứu trước của Ozturk et al. (2019). Từ đó, một số tính chất của các phép toán sẽ được trình bày và chứng minh.

Định nghĩa 2.6 Xét $\left(G_{1}, E\right)$ và $\left(G_{2}, E\right)$ là hai tập mềm trung tính trên tập vũ trụ $X$. Khi đó hợp của hai tập này được kí hiệu là $\left(G_{1}, E\right) \cup\left(G_{2}, E\right)=\left(G_{3}, E\right)$ và được xác định bởi:

$$
\left(G_{3}, E\right)=\left\{\left(e,\left\langle x, T_{G_{3}(e)}(x), I_{G_{3}(e)}(x), F_{G_{3}(e)}(x)\right\rangle: x \in X\right): e \in E\right\},
$$

trong đó

$$
\begin{aligned}
& T_{G_{3}(e)}(x)=T_{G_{1}(e)}(x)+T_{G_{2}(e)}(x)-T_{G_{1}(e)}(x) \cdot T_{G_{2}(e)}(x)=T_{G_{1}(e)}(x) \vee T_{G_{2}(e)}(x), \\
& I_{G_{3}(e)}(x)=I_{G_{1}(e)}(x)+I_{G_{2}(e)}(x)-I_{G_{1}(e)}(x) \cdot I_{G_{2}(e)}(x)=I_{G_{1}(e)}(x) \vee I_{G_{2}(e)}(x), \\
& F_{G_{3}(e)}(x)=F_{G_{1}(e)}(x) F_{G_{2}(e)}(x)=F_{G_{1}(e)}(x) \wedge F_{G_{2}(e)}(x)
\end{aligned}
$$


Định nghĩa 2.7 Cho $\left(G_{1}, E\right)$ và $\left(G_{2}, E\right)$ là hai tập mềm trung tính trên tập vũ trụX. Giao của hai tập này ký hiệu $\left(G_{1}, E\right) \cap\left(G_{2}, E\right)=\left(G_{3}, E\right)$ được xác định bởi:

$\left(G_{3}, E\right)=\left\{\left(e,\left\langle x, T_{G_{3}(e)}(x), I_{G_{3}(e)}(x), F_{G_{3}(e)}(x)\right\rangle: x \in X\right): e \in E\right\}$,

trong đó

$$
\begin{aligned}
& T_{G_{3}(e)}(x)=T_{G_{1}(e)}(x) \cdot T_{G_{2}(e)}(x)=T_{G_{1}(e)}(x) \wedge T_{G_{2}(e)}(x), \\
& I_{G_{3}(e)}(x)=I_{G_{1}(e)}(x) \cdot I_{G_{2}(e)}(x)=I_{G_{1}(e)}(x) \wedge I_{G_{2}(e)}(x), \\
& F_{G_{3}(e)}(x)=F_{G_{1}(e)}(x)+F_{G_{2}(e)}(x)-F_{G_{1}(e)}(x) \cdot F_{G_{2}(e)}(x)=F_{G_{1}(e)}(x) \vee F_{G_{2}(e)}(x) .
\end{aligned}
$$

Định nghĩa 2.8 Cho $\left(G_{1}, E\right)$ và $\left(G_{2}, E\right)$ là hai tập mềm trung tính trên tập vũ trụ $X$. Khi đó phép toán “ $\left(G_{1}, E\right)$ hiệu $\left(G_{2}, E\right)$ ", ký hiệu bởi $\left(G_{1}, E\right) \backslash\left(G_{2}, E\right)=\left(G_{3}, E\right)$, xác định bằng phép toán $\left(G_{1}, E\right) \cap\left(G_{2}, E\right)^{C}$, cụ thể như sau:

$$
\left(G_{3}, E\right)=\left\{\left(e,\left\langle x, T_{G_{3}(e)}(x), I_{G_{3}(e)}(x), F_{G_{3}(e)}(x)\right\rangle: x \in X\right): e \in E\right\}
$$

trong đó

$$
\begin{aligned}
& T_{G_{3}(e)}(x)=T_{G_{1}(e)}(x) \cdot F_{G_{2}(e)}(x)=T_{G_{1}(e)}(x) \wedge F_{G_{2}(e)}(x), \\
& I_{G_{3}(e)}(x)=I_{G_{1}(e)}(x)\left(1-I_{G_{2}(e)}(x)\right)=I_{G_{1}(e)}(x) \wedge\left(1-I_{G_{2}(e)}(x)\right) \\
& F_{G_{3}(e)}(x)=F_{G_{1}(e)}(x)+T_{G_{2}(e)}(x)-F_{G_{1}(e)}(x) \cdot T_{G_{2}(e)}(x)=F_{G_{1}(e)}(x) \vee T_{G_{2}(e)}(x) .
\end{aligned}
$$

Định nghĩa 2.9 Cho $\left\{\left(G_{1}, E\right) \mid i \in I\right\}$ là một họ các tập mềm trung tính trên tập vũ trụ $X$. Khi đó

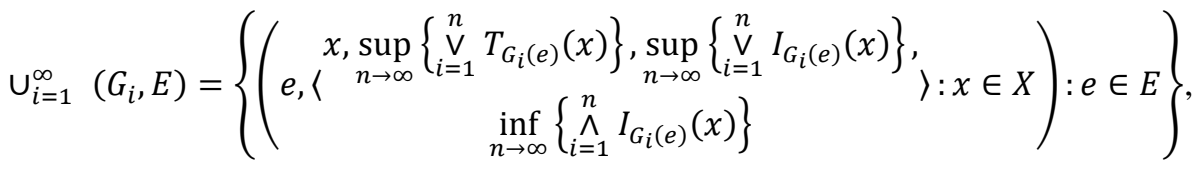

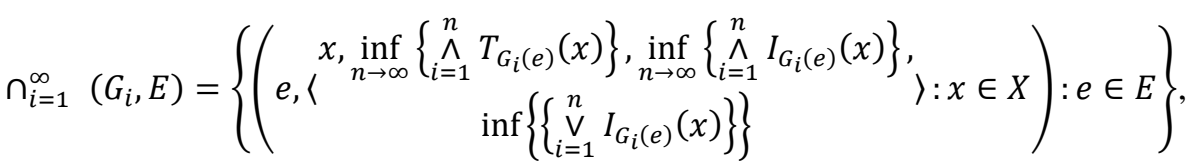

trong đó $\wedge, \vee$ là các phép toán được định nghĩa tương tự như trong các định nghĩa trên.

Định nghĩa 2.10 Cho $\left(G_{1}, E\right)$ và $\left(G_{2}, E\right)$ là tập mềm trung tính trênX. Khi đó, phép toán "AND" trên hai tập được ký hiệu bởi $\left(G_{1}, E\right) \wedge\left(G_{2}, E\right)=\left(G_{3}, E \times E\right)$ và được xác định như sau:

$$
\left(G_{3}, E \times E\right)=\left\{\left(\left(e_{1}, e_{2}\right),\left\langle x, T_{G_{3}(e)}(x), I_{G_{3}(e)}(x), F_{G_{3}(e)}(x)\right\rangle: x \in X\right):\left(e_{1}, e_{2}\right) \in E \times E\right\}
$$

trong đó

$$
\begin{gathered}
T_{G_{3}\left(e_{1}, e_{2}\right)}(x)=T_{G_{1}\left(e_{1}\right)}(x) \cdot T_{G_{2}\left(e_{2}\right)}(x), I_{G_{3}\left(e_{1}, e_{2}\right)}(x)=I_{G_{1}\left(e_{1}\right)}(x) \cdot I_{G_{2}\left(e_{2}\right)}(x), \\
F_{G_{3}\left(e_{1}, e_{2}\right)}(x)=F_{G_{1}\left(e_{1}\right)}(x)+F_{G_{2}\left(e_{2}\right)}(x)-F_{G_{1}\left(e_{1}\right)}(x) \cdot F_{G_{2}\left(e_{2}\right)}(x)
\end{gathered}
$$

Định nghĩa 2.11 Giả sử $\left(G_{1}, E\right)$ và $\left(G_{2}, E\right)$ là hai tập mềm trung tính trên $X$. Khi đó, phép toán "OR" trên hai tập này được ký hiệu bởi $\left(G_{1}, E\right) \vee\left(G_{2}, E\right)=\left(G_{3}, E \times E\right)$ và được xác định như sau:

$$
\left(G_{3}, E \times E\right)=\left\{\left(\left(e_{1}, e_{2}\right),\left\langle x, T_{G_{3}(e)}(x), I_{G_{3}(e)}(x), F_{G_{3}(e)}(x)\right\rangle: x \in X\right):\left(e_{1}, e_{2}\right) \in E \times E\right\}
$$

trong đó

$$
T_{G_{3}\left(e_{1}, e_{2}\right)}(x)=T_{G_{1}\left(e_{1}\right)}(x)+T_{G_{2}\left(e_{2}\right)}(x)-T_{G_{1}\left(e_{1}\right)}(x) \cdot T_{G_{2}\left(e_{2}\right)}(x), I_{G_{3}\left(e_{1}, e_{2}\right)}(x)=I_{G_{1}\left(e_{1}\right)}(x)+I_{G_{2}\left(e_{2}\right)}(x)-
$$
$I_{G_{1}\left(e_{1}\right)}(x) \cdot I_{G_{2}\left(e_{2}\right)}(x), F_{G_{3}\left(e_{1}, e_{2}\right)}(x)=F_{G_{1}\left(e_{1}\right)}(x) \cdot F_{G_{2}\left(e_{2}\right)}(x)$. 


\section{Định nghĩa 2.12}

Một tập mềm trung tính $(G, E)$ trên tập vũ trụ $X$ được gọi là tập mềm trung tính tuyệt đối nếu $T_{G(e)}(x)=$ $1 ; I_{G(e)}(x)=1 ; F_{G(e)}(x)=0$; với mọie $\in E, x \in X$. Khi đó $(G, E)$ được ký hiệu là1 $1_{(X, E)}$.

Hiển nhiên $0_{(X, E)}^{C}=1_{(X, E)}$ và1 $1_{(X, E)}^{C}=0_{(X, E)}$.

2. Tập mềm trung tính $(G, E)$ trên tập vũ trụ $X$ được gọi là tập mềm trung tính rỗng nếuT $T_{G(e)}(x)=$ $0 ; I_{G(e)}(x)=0 ; F_{G(e)}(x)=1$; với mọie $\in E, x \in X$. Khi đó $(G, E)$ được ký hiệu là $0_{(X, E)}$.

Mệnh đề 2.1 Cho $\left(G_{1}, E\right),\left(G_{2}, E\right)$ và $\left(G_{3}, E\right)$ là các tập mềm trung tính trên tập vũ trụ $X$. Khi đó

1. $\left(G_{1}, E\right) \cup\left[\left(G_{2}, E\right) \cup\left(G_{3}, E\right)\right]=\left[\left(G_{1}, E\right) \cup\left(G_{2}, E\right)\right] \cup\left(G_{3}, E\right)$ và

$\left(G_{1}, E\right) \cap\left[\left(G_{2}, E\right) \cap\left(G_{3}, E\right)\right]=\left[\left(G_{1}, E\right) \cap\left(G_{2}, E\right)\right] \cap\left(G_{3}, E\right)$.

2. $\left(G_{1}, E\right) \cup 0_{(X, E)}=\left(G_{1}, E\right) ;\left(G_{1}, E\right) \cap 0_{(X, E)}=0_{(X, E)}$.

3. $\left(G_{1}, E\right) \cup 1_{(X, E)}=1_{(X, E)} ;\left(G_{1}, E\right) \cap 1_{(X, E)}=\left(G_{1}, E\right)$.

Chúng minh. 1. Với mọi $e \in E$ và $x \in X$, ta xét vế phải,

$$
\begin{aligned}
& {\left[\left(G_{1}, E\right) \cup\left(G_{2}, E\right)\right] \cup\left(G_{3}, E\right)=\left\{\begin{array}{l}
\left\langle\begin{array}{l}
x, T_{G_{3}(e)}(x) \vee\left[T_{G_{1}(e)}(x)+T_{G_{2}(e)}(x)-T_{G_{1}(e)}(x) \cdot T_{G_{2}(e)}(x)\right], \\
I_{G_{3}(e)}(x) \vee\left[I_{G_{1}(e)}(x)+I_{G_{2}(e)}(x)-I_{G_{1}(e)}(x) \cdot I_{G_{2}(e)}(x)\right], \\
I_{G_{3}(e)}(x) \wedge\left[I_{G_{1}(e)}(x) I_{G_{2}(e)}(x)\right]
\end{array}\right\}
\end{array}\right\}} \\
& \left.=\left\{\begin{array}{l}
x, T_{G_{3}(e)}(x)+T_{G_{1}(e)}(x)+T_{G_{2}(e)}(x)-T_{G_{1}(e)}(x) T_{G_{2}(e)}(x)-T_{G_{3}(e)}(x) T_{G_{1}(e)}(x)-T_{G_{3}(e)}(x) T_{G_{2}(e)}(x) \\
+T_{G_{1}(e)}(x) T_{G_{2}(e)}(x) T_{G_{3}(e)}(x), \\
I_{G_{3}(e)}(x)+I_{G_{1}(e)}(x)+I_{G_{2}(e)}(x)-I_{G_{1}(e)}(x) I_{G_{2}(e)}(x)-I_{G_{3}(e)}(x) I_{G_{1}(e)}(x)-I_{G_{3}(e)}(x) I_{G_{2}(e)}(x) \\
+I_{G_{1}(e)}(x) I_{G_{2}(e)}(x) I_{G_{3}(e)}(x), \\
I_{G_{3}(e)}(x) I_{G_{1}(e)}(x) I_{G_{2}(e)}(x)
\end{array}\right)\right\}
\end{aligned}
$$

Ta xét vế trái:

$$
\left.\left(G_{1}, E\right) \cup\left[\left(G_{2}, E\right) \cup\left(G_{3}, E\right)\right]=\left\{\begin{array}{l}
x, T_{G_{1}(e)}(x) \vee\left[T_{G_{2}(e)}(x)+T_{G_{3}(e)}(x)-T_{G_{2}(e)}(x) T_{G_{3}(e)}(x)\right], \\
I_{G_{1}(e)}(x) \vee\left[I_{G_{2}(e)}(x)+I_{G_{3}(e)}(x)-I_{G_{2}(e)}(x) I_{G_{3}(e)}(x)\right], \\
F_{G_{1}(e)}(x) \wedge\left[F_{G_{2}(e)}(x) F_{G_{3}(e)}(x)\right]
\end{array}\right\}\right\}
$$

Vì vậy $\left(G_{1}, E\right) \cup\left[\left(G_{2}, E\right) \cup\left(G_{3}, E\right)\right]=\left[\left(G_{1}, E\right) \cup\left(G_{2}, E\right)\right] \cup\left(G_{3}, E\right)$.

Ta chứng minh tương tự với đẳng thức $\left(G_{1}, E\right) \cap\left[\left(G_{2}, E\right) \cap\left(G_{3}, E\right)\right]=\left[\left(G_{1}, E\right) \cap\left(G_{2}, E\right)\right] \cap\left(G_{3}, E\right)$.

2.Với mọi $e \in E$ và $x \in X$,

$$
\begin{aligned}
& \left(G_{1}, E\right) \cup 0_{(X, E)}=\left\{\left\langle x, T_{G_{1}(e)}(x)+0-T_{G_{1}(e)}(x) \cdot 0, I_{G_{1}(e)}(x)+0-I_{G_{1}(e)}(x) \cdot 0, F_{G_{1}(e)}(x) \cdot 1\right\rangle\right\} \\
& =\left\{\left\langle x, T_{G_{1}(e)}(x), I_{G_{1}(e)}(x), F_{G_{1}(e)}(x)\right\rangle\right\}=\left(G_{1}, E\right),
\end{aligned}
$$




$$
\left(G_{1}, E\right) \cap 0_{(X, E)}=\left\{\left\langle x, T_{G_{1}(e)}(x) \cdot 0, I_{G_{1}(e)}(x) \cdot 0, F_{G_{1}(e)}(x)+1-F_{G_{1}(e)}(x) \cdot 1\right\rangle\right\}=\{\langle x, 0,0,1\rangle\}=0_{(X, E)} .
$$

3.Với mọi $e \in E$ vàx $\in X$,

$$
\begin{aligned}
\left(G_{1}, E\right) \cup 1_{(X, E)} & =\left\{\left\langle x, T_{G_{1}(e)}(x)+1-T_{G_{1}(e)}(x) \cdot 1, I_{G_{1}(e)}(x)+1-I_{G_{1}(e)}(x) \cdot 1, F_{G_{1}(e)}(x) \cdot 0\right\rangle\right\} \\
& =\{\langle x, 1,1,0\rangle\}=1_{(X, E)}, \\
\left(G_{1}, E\right) \cap 1_{(X, E)} & =\left\{\left\langle x, T_{G_{1}(e)}(x) \cdot 1, I_{G_{1}(e)}(x) \cdot 1, F_{G_{1}(e)}(x)+0-F_{G_{1}(e)}(x) \cdot 0\right\rangle\right\} \\
& =\left\{\left\langle x, T_{G_{1}(e)}(x), I_{G_{1}(e)}(x), F_{G_{1}(e)}(x)\right\rangle\right\}=\left(G_{1}, E\right) .
\end{aligned}
$$

Chú ý 2.1 Xét một cách tổng quát, các đẳng thức:

$$
\begin{aligned}
& \left(G_{1}, E\right) \cup\left[\left(G_{2}, E\right) \cap\left(G_{3}, E\right)\right]=\left[\left(G_{1}, E\right) \cup\left(G_{2}, E\right)\right] \cap\left[\left(G_{1}, E\right) \cup\left(G_{3}, E\right)\right], \\
& \left(G_{1}, E\right) \cap\left[\left(G_{2}, E\right) \cup\left(G_{3}, E\right)\right]=\left[\left(G_{1}, E\right) \cap\left(G_{2}, E\right)\right] \cup\left[\left(G_{1}, E\right) \cap\left(G_{3}, E\right)\right],
\end{aligned}
$$

không đúng với phép toán hợp, giao mới được định nghĩa trên tập mềm trung tính.

Mệnh đề 2.2 Cho $\left(G_{1}, E\right),\left(G_{2}, E\right)$ là hai tập mềm trung tính trên tập vũ trụX. Khi đó,

$\left[\left(G_{1}, E\right) \cup\left(G_{2}, E\right)\right]^{C}=\left(G_{1}, E\right)^{C} \cap\left(G_{2}, E\right)^{C} ;\left[\left(G_{1}, E\right) \cap\left(G_{2}, E\right)\right]^{C}=\left(G_{1}, E\right)^{C} \cup\left(G_{2}, E\right)^{C}$

Chúng minh. Với mọi $e \in E$ và $x \in X$, ta có

$$
\left.\left[\left(G_{1}, E\right) \cup\left(G_{2}, E\right)\right]^{C}=\left\{\begin{array}{l}
x, F_{G_{1}(e)}(x) F_{G_{2}(e)}(x), 1-I_{G_{1}(e)}(x)-I_{G_{2}(e)}(x)+I_{G_{1}(e)}(x) I_{G_{2}(e)}(x), \\
T_{G_{1}(e)}(x)+T_{G_{2}(e)}(x)-T_{G_{1}(e)}(x) T_{G_{2}(e)}(x)
\end{array}\right)\right\}
$$

Mặt khác,

$$
\begin{aligned}
& \left.\left(G_{1}, E\right)^{C} \cap\left(G_{2}, E\right)^{C}=\left\{\begin{array}{l}
x, F_{G_{1}(e)}(x) F_{G_{2}(e)}(x),\left[1-I_{G_{1}(e)}(x)\right]\left[1-I_{G_{2}(e)}(x)\right], \\
T_{G_{1}(e)}(x)+T_{G_{2}(e)}(x)-T_{G_{1}(e)}(x) T_{G_{2}(e)}(x)
\end{array}\right)\right\} \\
& =\left\{\left\langle\begin{array}{l}
x, F_{G_{1}(e)}(x) F_{G_{2}(e)}(x), 1-I_{G_{1}(e)}(x)-I_{G_{2}(e)}(x)+I_{G_{1}(e)}(x) I_{G_{2}(e)}(x), \\
T_{G_{1}(e)}(x)+T_{G_{2}(e)}(x)-T_{G_{1}(e)}(x) T_{G_{2}(e)}(x)
\end{array}\right)\right\}
\end{aligned}
$$

Vì vậy, $\left[\left(G_{1}, E\right) \cup\left(G_{2}, E\right)\right]^{C}=\left(G_{1}, E\right)^{C} \cap\left(G_{2}, E\right)^{C}$.

Ta chứng minh tương tự với đẳng thức $\left[\left(G_{1}, E\right) \cap\left(G_{2}, E\right)\right]^{C}=\left(G_{1}, E\right)^{C} \cup\left(G_{2}, E\right)^{C}$.

Mệnh đề 2.3 Cho $\left(G_{1}, E\right),\left(G_{2}, E\right)$ là hai tập mềm trung tính trên tập vũ trụX. Khi đó,

$\left[\left(G_{1}, E\right) \vee\left(G_{2}, E\right)\right]^{C}=\left(G_{1}, E\right)^{C} \wedge\left(G_{2}, E\right)^{C} ;\left[\left(G_{1}, E\right) \wedge\left(G_{2}, E\right)\right]^{C}=\left(G_{1}, E\right)^{C} \vee\left(G_{2}, E\right)^{C}$.

Chúng minh. Với mọi $e_{1}, e_{2} \in E$ vàx $\in X$, ta có

$$
\left.\left[\left(G_{1}, E\right) \vee\left(G_{2}, E\right)\right]^{C}=\left\{\begin{array}{l}
x, F_{G_{1}\left(e_{1}\right)}(x) F_{G_{2}\left(e_{2}\right)}(x), 1-I_{G_{1}\left(e_{1}\right)}(x)-I_{G_{2}\left(e_{2}\right)}(x)+I_{G_{1}\left(e_{1}\right)}(x) I_{G_{2}\left(e_{2}\right)}(x), \\
T_{G_{1}\left(e_{1}\right)}(x)+T_{G_{2}\left(e_{2}\right)}(x)-T_{G_{1}\left(e_{1}\right)}(x) T_{G_{2}\left(e_{2}\right)}(x)
\end{array}\right)\right\} .
$$


Mặt khác,

$$
\begin{aligned}
& \left.\left(G_{1}, E\right)^{C} \wedge\left(G_{2}, E\right)^{C}=\left\{\begin{array}{l}
x, F_{G_{1}\left(e_{1}\right)}(x) F_{G_{2}\left(e_{2}\right)}(x),\left[1-I_{G_{1}\left(e_{1}\right)}(x)\right]\left[1-I_{G_{2}\left(e_{2}\right)}(x)\right], \\
T_{G_{1}\left(e_{1}\right)}(x)+T_{G_{2}\left(e_{2}\right)}(x)-T_{G_{1}\left(e_{1}\right)}(x) T_{G_{2}\left(e_{2}\right)}(x)
\end{array}\right)\right\} \\
& \left.=\left\{\begin{array}{l}
x, F_{G_{1}\left(e_{1}\right)}(x) F_{G_{2}\left(e_{2}\right)}(x), 1-I_{G_{1}\left(e_{1}\right)}(x)-I_{G_{2}\left(e_{2}\right)}(x)+I_{G_{1}\left(e_{1}\right)}(x) I_{G_{2}\left(e_{2}\right)}(x), \\
T_{G_{1}\left(e_{1}\right)}(x)+T_{G_{2}\left(e_{2}\right)}(x)-T_{G_{1}\left(e_{1}\right)}(x) T_{G_{2}\left(e_{2}\right)}(x)
\end{array}\right)\right\}
\end{aligned}
$$

Vì vậy, $\left[\left(G_{1}, E\right) \vee\left(G_{2}, E\right)\right]^{C}=\left(G_{1}, E\right)^{C} \wedge\left(G_{2}, E\right)^{C}$.

Ta chứng minh tương tự đối với đẳng thức $\left[\left(G_{1}, E\right) \wedge\left(G_{2}, E\right)\right]^{C}=\left(G_{1}, E\right)^{C} \vee\left(G_{2}, E\right)^{C}$.

\section{KẾT QUẢ VÀ THỰC HIỆN}

Ở mục này, tác giả xây dựng không gian tôpô mềm trung tính dựa trên các định nghĩa mới về phép hợp, phép giao, tập mềm trung tính tuyệt đối, tập mềm trung tính rỗng đã được đưa ra ở phần trước. Từ đó, bài viết phát biểu và chứng minh các mệnh đề và định lý trên không gian tôpô đã xây dựng. Hầu hết các định lý và mệnh đề đều có cách chứng minh tương tự như trong bài báo của Ozturk et al. (2019).

Định nghĩa 3.1 Giả sử $N S S(X, E)$ là tập chứa tất cả các tập mềm trung tính trên tập vũ trụ $X$ và $\tau$ NSS $\subset$

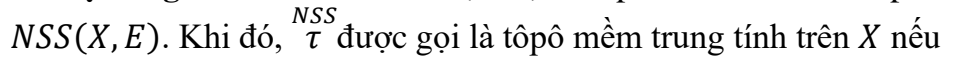

1. $0_{(X, E)}$ và $1_{(X, E)}$ đều là phần tử của $\tau$ $\tau$.

2.Hợp của một họ bất kỳ các tập mềm trung tính trong $\stackrel{N S S}{\tau}$ đều nằm trong $\tau$ $\tau$.

3.Giao hữu hạn các tập mềm trung tính trong ${ }^{N S S}$ thì thuộc ${ }^{N S S}$.

Khi đó $(X, \stackrel{N S S}{\tau}, E)$ là tập mềm trung tính trênX. Mỗi phần tử của ${ }_{\tau}^{N S S}$ được gọi là tập mở trong ${ }^{N S S}$.

Định nghĩa 3.2 Cho $(X, \stackrel{N S S}{\tau}, E)$ là không gian tôpô mềm trung tính trên $X$ và $(G, E)$ là một tập mềm trung tính trên $X$. Khi đó, $(G, E)$ được gọi là tập đóng thuộc ${ }_{\tau}^{N S S}$ khi và chỉ khi phần bù của nó là tập mở trong ${ }^{N S S}$.

Mệnh đề 3.1 Giả sử $(X, \stackrel{N S S}{\tau}, E)$ là không gian tôpô trên $X$. Khi đó

1. $0_{(X, E)}$ và $1_{(X, E)}$ là tập mềm trung tính đóng trên $X$.

2.Giao của một họ bất kỳ các tập mềm trung tính đóng trong ${ }_{\tau}^{N S S}$ là tập mềm trung tính đóng trong $\tau$ $\tau$.

3.Hợp hữu hạn của các tập trung tính mềm đóng trong $\stackrel{N S S}{\tau}$ là một tập mềm trung tính đóng trong $\stackrel{N S S}{\tau}$.

\section{Chứng minh.}

1. $0_{(X, E)}^{C}=1_{(X, E)} \in \stackrel{N S S}{\tau} ; 1_{(X, E)}^{C}=0_{(X, E)} \in \stackrel{N S S}{\tau}$

2.Giả sử $\left\{\left(G_{i}, E\right) \mid i \in I\right\}$ là một họ các tập mềm trung tính đóng trên $X$. Do đó $\left(G_{i}, E\right)^{C} \in{ }_{\tau}^{N S S}$ với mọi $i \in I$ nên $\left[\cap_{i \in I}\left(G_{i}, E\right)\right]^{C}=\cup_{i \in I}\left(G_{i}, E\right)^{C} \in{ }^{N S S}$.Vì vậy $\cap_{i \in I} \quad\left(G_{i}, E\right)$ là tập mềm trung tính đóng trên $X$. Điều này được suy ra từ định nghĩa của không gian tôpô mềm trung tính trên $X$ và mệnh đề 2.2.

3.Cho $\left(G_{1}, E\right),\left(G_{2}, E\right)$ là hai tập mềm trung tính đóng trênX. Khi đó, $\left(G_{1}, E\right)^{C} \in \stackrel{N S S}{\tau},\left(G_{2}, E\right)^{C} \in \stackrel{N S S}{\tau}$. Vì vậy, $\left[\left(G_{1}, E\right) \cup\left(G_{2}, E\right)\right]^{C}=\left(G_{1}, E\right)^{c} \cap\left(G_{2}, E\right)^{c} \in \stackrel{N S S}{\tau}$ do đó ta suy ra $\left(G_{1}, E\right) \cup\left(G_{2}, E\right)$ là tập mềm trung tính đóng trên $X$. 
Định nghĩa 3.3 Cho $N S S(X, E)$ là tập chứa tất cả các tập mềm trung tính trênX.

1. Nếu $\stackrel{N S S}{\tau}=\left\{0_{(X, E)}, 1_{(X, E)}\right\}$ thì $\stackrel{N S S}{\tau}$ được gọi là tôpô rời rạc mềm trung tính và khi đó $\left(X,{ }^{N S S}, E\right)$ được gọi là không gian tôpô không rời rạc mềm trung tính trên $X$.

2.Nếu $\stackrel{N S S}{\tau}=N S S(X, E)$, thì $\stackrel{N S S}{\tau}$ được gọi là tôpô rời rạc và $(X, \stackrel{N S S}{\tau}, E)$ được gọi là không gian tôpô rời rạc trên $X$.

Mệnh đề 3.2 Giả sử $\left(X,{ }^{N S S} \tau_{1}, E\right)$ và $\left(X, \stackrel{N S S}{\tau_{2}}, E\right)$ là hai không gian tôpô mềm trung tính trên tập vũ trụ $X$. Khi đó, ta cũng có $\left(X, \tau_{1}^{N S S} \cap \stackrel{N S S}{\tau_{2}}, E\right)$ là một không gian tôpô mềm trung tính trên $X$.

Chứng minh.

$$
0_{(X, E)}, 1_{(X, E)} \in \stackrel{N S S}{\tau_{1}} ; 0_{(X, E)}, 1_{(X, E)} \in \stackrel{N S S}{\tau_{2}} \Rightarrow 0_{(X, E)}, 1_{(X, E)} \in \stackrel{N S S}{\tau_{1}} \cap \stackrel{N S S}{\tau_{2}}
$$

Cho $\left\{\left(G_{i}, E\right) \mid i \in I\right\}$ là một họ các tập mềm trung tính thuộc $\tau_{1}^{N S S} \cap{ }^{N S S}$. Ta có $\left(G_{i}, E\right) \in \tau_{1}$ và $\left(G_{i}, E\right) \in \tau_{2}^{N S S} \quad$ với mọi $i \in I$. Vì vậy $\quad \cup_{i \in I}\left(G_{i}, E\right) \in \tau_{1}^{N S S} ; \cup_{i \in I}\left(G_{i}, E\right) \in \tau_{2}$. Do $\quad$ đó $\quad$ ta $\quad$ suy ra $\cup_{i \in I}\left(G_{i}, E\right) \in \stackrel{N S S}{\tau_{1}} \cap \tau_{2}^{N S S}$.

Giả sử ta lấy $\left(G_{1}, E\right) \in \tau_{1}^{N S S} \cap \tau_{2}^{N S S} ;\left(G_{2}, E\right) \in \tau_{1}^{N S S} \cap \tau_{2}^{N S S}$. Khi đó $\quad\left(G_{1}, E\right) \in \tau_{1}^{N S S} ;\left(G_{2}, E\right) \in \tau_{1} \quad$ và $\left(G_{1}, E\right) \in \tau_{2} ;\left(G_{2}, E\right) \in \tau_{2}$, nên

$$
\left(G_{1}, E\right) \cap\left(G_{2}, E\right) \in \tau_{1}^{N S S} ;\left(G_{1}, E\right) \cap\left(G_{2}, E\right) \in \tau_{2}^{N S S}
$$

Do đó $\left(G_{1}, E\right) \cap\left(G_{2}, E\right) \in \tau_{1}^{N S S} \cap \tau_{2}^{N S S}$.

Từ ba điều trên ta chứng minh được $\left(X, \tau_{1}^{N S S} \cap \tau_{2}, E\right)$ là một không gian tôpô mềm trung tính trên $X$.

Chú ý 3.1 Hợp của hai không gian tôpô mềm trung tính trên $X$ không chắc là không gian tôpô mềm trung tính trên $X$.

Ví dụ 3.1 Cho $X=\left\{x_{1}, x_{2}, x_{3}\right\}$ là tập vũ trụ, $E=\left\{e_{1}, e_{2}\right\}$ là tập các tham số. Xét $\tau_{1}^{\text {NSS }}=\left\{0_{(X, E)}, 1_{(X, E)},\left(G_{1}, E\right),\left(G_{2}, E\right)\right\} \quad$ và ${ }^{\text {NSS }}=\left\{0_{(X, E)}, 1_{(X, E)},\left(G_{3}, E\right)\right\}$ là hai tôpô mềm trung tính trên $X$, trong đó $\left(G_{1}, E\right),\left(G_{2}, E\right)$ và $\left(G_{3}, E\right)$ được xác định như sau:

$$
\begin{aligned}
& \left(G_{1}, E\right)=\left\{\begin{array}{l}
e_{1}=\left\{\left\langle x_{1}, 1,0,1\right\rangle,\left\langle x_{2}, 1,0,1\right\rangle\right\}, \\
\left.e_{2}=\left\{\left\langle x_{1}, 0,1,0\right\rangle,\left\langle x_{2}, 0,1,0\right\rangle\right\}\right\},
\end{array}\right. \\
& \left(G_{2}, E\right)=\left\{\begin{array}{l}
e_{1}=\left\{\left\langle x_{1}, 0,1,0\right\rangle,\left\langle x_{2}, 0,1,0\right\rangle\right\}, \\
e_{2}=\left\{\left\langle x_{1}, 1,0,1\right\rangle,\left\langle x_{2}, 1,0,1\right\rangle\right\}
\end{array}\right\},
\end{aligned}
$$




$$
\left(G_{3}, E\right)=\left\{\begin{array}{l}
e_{1}=\left\{\left\langle x_{1}, 1,0,1\right\rangle,\left\langle x_{2}, 1,0,1\right\rangle\right\} \\
e_{2}=\left\{\left\langle x_{1}, 1,0,1\right\rangle,\left\langle x_{2}, 1,0,1\right\rangle\right\}
\end{array}\right\}
$$

$\left(G_{1}, E\right) \cup\left(G_{3}, E\right)=$

Vì $\left\{\begin{array}{l}e_{1}=\left\{\left\langle x_{1}, 1,0,1\right\rangle,\left\langle x_{2}, 1,0,1\right\rangle\right\}, \\ e_{2}=\left\{\left\langle x_{1}, 1,1,0\right\rangle,\left\langle x_{2}, 1,1,0\right\rangle\right\}\end{array}\right\} \notin \tau_{1} \cup \tau_{2} \quad$ nSS nên $\tau_{1} \cup \tau_{2} \cup \tau_{2}$ không phải là tôpô mè̀m trung tính trên $X$

Mệnh đề 3.3 Xét $(X, \stackrel{N S S}{\tau}, E)$ là không gian tôpô mềm trung tính trên $X$, trong đó

$$
\begin{aligned}
& \stackrel{N S S}{\tau}=\left\{\left(G_{i}, E\right):\left(G_{i}, E\right) \in N S S(X, E)\right\} \quad \text { với }\left(G_{i}, e\right)=\left\{\left\langle x, T_{G_{i}(e)}(x), I_{G_{i}(e)}(x), F_{G_{i}(e)}(x)\right\rangle: x \in X\right\} . \text { Khi } \\
& =\left\{\left[e, G_{i}(e)\right]_{e \in E}:\left(G_{i}, E\right) \in N S S(X, E)\right\}
\end{aligned}
$$

đó, không gian này cảm sinh

$$
\tau_{1}=\left\{\left[T_{G_{i}(e)}(X)\right]_{e \in E}\right\}, \tau_{2}=\left\{\left[I_{G_{i}(e)}(X)\right]_{e \in E}\right\}, \tau_{3}=\left\{\left[F_{G_{i}(e)}(X)\right]_{e \in E}^{C}\right\},
$$

là các tôpô mềm mờ trên $X$.

\section{Chứng minh.}

1.Hiển nhiên.

2.Lấy họ tập mềm trung tính $\left\{\left(G_{i}, E\right)\right\}_{i=1}^{\infty} \in \tau$ NSS . Ta có,

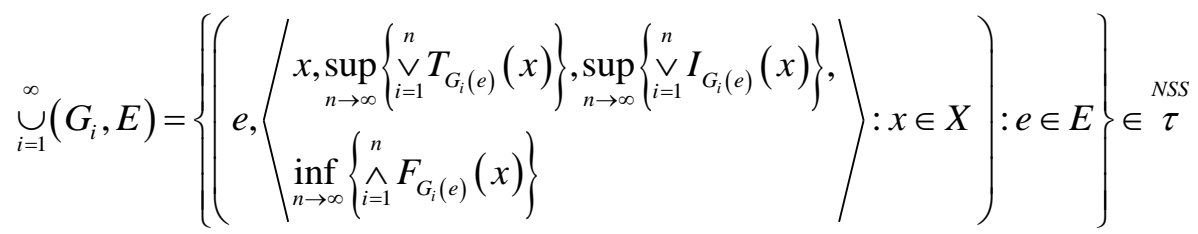

Vì vậy,

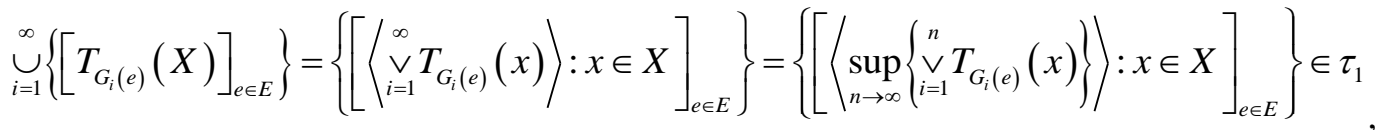

$$
\begin{aligned}
& \bigcup_{i=1}^{\infty}\left\{\left[I_{G_{i}(e)}(X)\right]_{e \in E}\right\}=\left\{\left[\left\langle\sum_{i=1}^{\infty} I_{G_{i}(e)}(x)\right\rangle: x \in X\right]_{e \in E}\right\}=\left\{\left[\left\langle\sup _{n \rightarrow \infty}\left\{\underset{i=1}{\vee} I_{G_{i}(e)}(x)\right\}\right): x \in X\right]_{e \in E}\right\} \in \tau_{2} \text {. } \\
& \bigcup_{i=1}^{\infty}\left\{\left[\left[I_{G_{i}(e)}(X)\right]^{C}\right]_{e \in E}\right\}=\left\{\left[\left\langle\left\{\underset{i=1}{\infty}\left(1-F_{G_{i}(e)}(x)\right)\right\}\right): x \in X\right]_{e \in E}\right\}=\left\{\left[\left\langle\sup _{n \rightarrow \infty}\left\{\underset{i=1}{\vee}\left(1-F_{G_{i}(e)}(x)\right)\right\}\right): x \in X\right]_{e \in E}\right\} \\
& =\left\{\left[\left\langle\sup _{n \rightarrow \infty}\left\{1-\hat{\imath}_{i=1}^{n} F_{G_{i}(e)}(x)\right\}\right\rangle: x \in X\right]_{e \in E}\right\}=\left\{\left[\left\langle 1-\inf _{n \rightarrow \infty}\left\{\hat{\wedge}_{i=1}^{n} F_{G_{i}(e)}(x)\right\}\right\rangle: x \in X\right]_{e \in E}\right\} \in \tau_{3} \\
& \text { 3.Ta có }\left(G_{1}, E\right) \cap\left(G_{2}, E\right) \in \tau^{N S S}
\end{aligned}
$$




$$
\left.\left(G_{1}, E\right) \cap\left(G_{2}, E\right)=\left\{\left(\begin{array}{l}
x, T_{G_{1}(e)}(x) T_{G_{2}(e)}(x), \\
I_{G_{1}(e)}(x) I_{G_{2}(e)}(x), \\
F_{G_{1}(e)}(x)+F_{G_{2}(e)}(x)-F_{G_{1}(e)}(x) F_{G_{2}(e)}(x)
\end{array}\right): x \in X\right): e \in E\right\}
$$

Vì vậy,

$$
\begin{aligned}
& \left\{\left[\left\langle T_{G_{1}(e)}(x) T_{G_{2}(e)}(x)\right\rangle: x \in X\right]_{e \in E}\right\}=\left\{\left[T_{G_{1}(e)}(X)\right]_{e \in E}\right\} \cap\left\{\left[T_{G_{2}(e)}(X)\right]_{e \in E}\right\} \in \tau_{1}, \\
& \left\{\left[\left\langle I_{G_{1}(e)}(x) I_{G_{2}(e)}(x)\right\rangle: x \in X\right]_{e \in E}\right\}=\left\{\left[I_{G_{1}(e)}(X)\right]_{e \in E}\right\} \cap\left\{\left[I_{G_{2}(e)}(X)\right]_{e \in E}\right\} \in \tau_{2}, \\
& \left\{\left[\left\langle F_{G_{1}(e)}(x)+F_{G_{2}(e)}(x)-F_{G_{1}(e)}(x) F_{G_{2}(e)}(x)\right\rangle: x \in X\right]_{e \in E}\right\} \\
& =\left\{\left[\left\langle 1-F_{G_{1}(e)}(x)-F_{G_{2}(e)}(x)+F_{G_{1}(e)}(x) F_{G_{2}(e)}(x)\right\rangle: x \in X\right]_{e \in E}\right\} \\
& =\left\{\left[\left\langle\left(1-F_{G_{1}(e)}(x)\right)\left(1-F_{G_{2}(e)}(x)\right)\right\rangle: x \in X\right]_{e \in E}\right\} \\
& =\left\{\left[F_{G_{1}(e)}(X)\right]_{e \in E}^{C}\right\} \cap\left\{\left[F_{G_{2}(e)}(X)\right]_{e \in E}^{C}\right\} \in \tau_{3} .
\end{aligned}
$$

Chú ý 3.2 Chiều ngược lại của mệnh đề trên không đúng khi xét tổng quát.

Ví dụ 3.2 Cho $X=\left\{x_{1}, x_{2}\right\}$ là tập vũ trụ, $E=\left\{e_{1}, e_{2}\right\}$ là tập các tham số. Các tập mềm trung tính $\left(G_{1}, E\right),\left(G_{2}, E\right),\left(G_{3}, E\right)$ và $\left(G_{4}, E\right)$ được mô tả cụ thể như sau:

$$
\begin{aligned}
& \left(G_{1}, E\right)=\left\{\begin{array}{l}
e_{1}=\left\{\left\langle x_{1}, 1,0,0\right\rangle,\left\langle x_{2}, 0,1,1\right\rangle\right\}, \\
e_{2}=\left\{\left\langle x_{1}, 0,1,1\right\rangle,\left\langle x_{2}, 1,0,0\right\rangle\right\}
\end{array}\right\}, \\
& \left(G_{2}, E\right)=\left\{\begin{array}{l}
e_{1}=\left\{\left\langle x_{1}, 0,1,1\right\rangle,\left\langle x_{2}, 1,0,0\right\rangle\right\}, \\
e_{2}=\left\{\left\langle x_{1}, 1,0,0\right\rangle,\left\langle x_{2}, 0,1,1\right\rangle\right\}
\end{array}\right\}, \\
& \left(G_{3}, E\right)=\left\{\begin{array}{l}
e_{1}=\left\{\left\langle x_{1}, 1,1,0\right\rangle,\left\langle x_{2}, 1,1,0\right\rangle\right\}, \\
e_{2}=\left\{\left\langle x_{1}, 0,0,1\right\rangle,\left\langle x_{2}, 1,1,0\right\rangle\right\}
\end{array}\right\}, \\
& \left(G_{4}, E\right)=\left\{\begin{array}{l}
e_{1}=\left\{\left\langle x_{1}, 0,0,1\right\rangle,\left\langle x_{2}, 1,1,0\right\rangle\right\}, \\
e_{2}=\left\{\left\langle x_{1}, 0,0,1\right\rangle,\left\langle x_{2}, 0,0,1\right\rangle\right\}
\end{array}\right\},
\end{aligned}
$$

Khi đó,

$$
\begin{gathered}
\tau_{1}=\left\{\begin{array}{r}
\left\{(0,0)_{e_{1}},(0,0)_{e_{2}}\right\},\left\{(1,1)_{e_{1}},(1,1)_{e_{2}}\right\},\left\{(1,0)_{e_{1}},(0,1)_{e_{2}}\right\}, \\
\left\{(0,1)_{e_{1}},(1,0)_{e_{2}}\right\},\left\{(1,1)_{e_{1}},(0,1)_{e_{2}}\right\},\left\{(0,1)_{e_{1}},(0,0)_{e_{2}}\right\}
\end{array}\right\}, \tau_{2} \\
=\left\{\begin{array}{l}
\left\{(0,0)_{e_{1}},(0,0)_{e_{2}}\right\},\left\{(1,1)_{e_{1}},(1,1)_{e_{2}}\right\},\left\{(0,1)_{e_{1}},(1,0)_{e_{2}}\right\}, \\
\left\{(1,0)_{e_{1}},(0,1)_{e_{2}}\right\},\left\{(1,1)_{e_{1}},(0,1)_{e_{2}}\right\},\left\{(0,1)_{e_{1}},(0,0)_{e_{2}}\right\}
\end{array}\right\}, \\
\tau_{3}=\left\{\begin{array}{l}
\left\{(0,0)_{e_{1}},(0,0)_{e_{2}}\right\},\left\{(1,1)_{e_{1}},(1,1)_{e_{2}}\right\},\left\{(0,1)_{e_{1}},(1,0)_{e_{2}}\right\}, \\
\left\{(1,0)_{e_{1}},(0,1)_{e_{2}}\right\},\left\{(0,0)_{e_{1}},(1,0)_{e_{2}}\right\},\left\{(1,0)_{e_{1}},(1,1)_{e_{2}}\right\}
\end{array}\right\},
\end{gathered}
$$

là các tôpô mềm mờ trên $X$. Trong khi đó ${ }^{N S S}=\left\{0_{(X, E)}, 1_{(X, E)},\left(G_{1}, E\right),\left(G_{2}, E\right),\left(G_{3}, E\right),\left(G_{4}, E\right)\right\}$ không phải là tôpô mềm trung tính trên $X$ vì $\left(G_{1}, E\right) \cup\left(G_{3}, E\right) \notin \stackrel{N S S}{\tau}$.

Mệnh đề 3.4 Cho $(X, \stackrel{N S S}{\tau}, E)$ là không gian tôpô mềm trung tính trên tập vũ trụ $X$. Khi đó 


$$
\tau_{1 e}=\left\{\left[T_{G_{i}(e)}(X)\right]:\left(G_{i}, E\right) \in \stackrel{N S S}{\tau}\right\}, \tau_{2 e}=\left\{\left[I_{G_{i}(e)}(X)\right]:\left(G_{i}, E\right) \in \stackrel{N S S}{\tau}\right\}, \tau_{3 e}=\left\{\left[F_{G_{i}(e)}(X)\right]^{C}:\left(G_{i}, E\right) \in \stackrel{N S S}{\tau}\right\}
$$

với mỗi $e \in E$, xác định các tôpô mờ trên $X$.

Chúng minh. Mệnh đề trên có thể được suy ra từ mệnh đề 3.3 .

Chú ý 3.3 Xét một cách tổng quát, chiều đảo của mệnh đề 3.4 không đúng. Chúng ta có thể xét ví dụ 3.3 phía dưới.

Ví dụ 3.3 Cho $X=\left\{x_{1}, x_{2}\right\}$ là tập vũ trụ, $E=\left\{e_{1}, e_{2}\right\}$ là tập các tham số. Các tập mềm trung tính $\left(G_{1}, E\right),\left(G_{2}, E\right),\left(G_{3}, E\right)$ và $\left(G_{4}, E\right)$ được mô tả như ví dụ 4.2 :

$$
\begin{aligned}
& \left(G_{1}, E\right)=\left\{\begin{array}{l}
e_{1}=\left\{\left\langle x_{1}, 1,0,0\right\rangle,\left\langle x_{2}, 0,1,1\right\rangle\right\}, \\
e_{2}=\left\{\left\langle x_{1}, 0,1,1\right\rangle,\left\langle x_{2}, 1,0,0\right\rangle\right\}
\end{array}\right\}, \\
& \left(G_{2}, E\right)=\left\{\begin{array}{l}
e_{1}=\left\{\left\langle x_{1}, 0,1,1\right\rangle,\left\langle x_{2}, 1,0,0\right\rangle\right\}, \\
e_{2}=\left\{\left\langle x_{1}, 1,0,0\right\rangle,\left\langle x_{2}, 0,1,1\right\rangle\right\}
\end{array}\right\}, \\
& \left(G_{3}, E\right)=\left\{\begin{array}{l}
e_{1}=\left\{\left\langle x_{1}, 1,1,0\right\rangle,\left\langle x_{2}, 1,1,0\right\rangle\right\}, \\
e_{2}=\left\{\left\langle x_{1}, 0,0,1\right\rangle,\left\langle x_{2}, 1,1,0\right\rangle\right\}
\end{array}\right\}, \\
& \left(G_{4}, E\right)=\left\{\begin{array}{l}
e_{1}=\left\{\left\langle x_{1}, 0,0,1\right\rangle,\left\langle x_{2}, 1,1,0\right\rangle\right\}, \\
e_{2}=\left\{\left\langle x_{1}, 0,0,1\right\rangle,\left\langle x_{2}, 0,0,1\right\rangle\right\}
\end{array}\right\},
\end{aligned}
$$

Ta có,

$$
\begin{aligned}
& \tau_{1 e_{1}}=\{(0,0),(1,1),(1,0),(0,1),(1,1),(0,1)\}=\{(0,0),(1,1),(1,0),(0,1)\} \\
& \tau_{2 e_{1}}=\{(0,0),(1,1),(0,1),(1,0),(1,1),(0,1)\}=\{(0,0),(1,1),(0,1),(1,0)\} \\
& \tau_{3 e_{1}}=\{(0,0),(1,1),(0,1),(1,0),(0,0),(1,0)\}=\{(0,0),(1,1),(0,1),(1,0)\}
\end{aligned}
$$

là các tôpô mờ trên $X$. Mặt khác, rõ ràng $\stackrel{N S S}{\tau}=\left\{0_{(X, E)}, 1_{(X, E)},\left(G_{1}, E\right),\left(G_{2}, E\right),\left(G_{3}, E\right),\left(G_{4}, E\right)\right\}$ không là tôpô mềm trung tính trên $X$ vì $\left(G_{1}, E\right) \cup\left(G_{3}, E\right) \notin \stackrel{N S S}{\tau}$.

\section{KẾT LUẬN}

Bài báo đã đưa ra các phép toán mới, khác với các phép toán trên tập mềm trung tính trong nghiên cứu của Ozturk et al. (2019). Từ đó, tác giả xây dựng không gian tôpô mềm trung tính từ các phép toán này với tiêu chí vẫn đảm bảo được mối quan hệ giữa không gian tôpô mềm trung tính và không gian tôpô mờ cũng như không gian tôpô mềm mờ. Ngoài ra, điều tác giả muốn chứng minh ở đây là có thể xây dựng được phép toán khác trên tập mềm trung tính để nó trở thành một không gian tôpô. Và không gian tôpô này khi quy chiếu về các không gian thành phần vẫn đảm bảo là không gian tôpô trên các phép toán đã xây dựng. Từ đó, tác giả hi vọng có thể xây dựng được các phép toán tổng quát đảm bảo điều kiện trên để phục vụ các nghiên cứu về tập mềm trung tính.

\section{TÀI LIỆU THAM KHẢO}

Bera, T., \& Mahapatra, N.K. (2017). Introduction to neutrosophic soft topological space. Opsearch,
54(4), 841-867. https://doi.org/10.1007/s12597017-0308-7

Deli, I., \& Broumi, S. (2015). Neutrosophic soft relations and some properties. Annals of Fuzzy Mathematics and Informatics, 9(1), 169-182. http://doi.org/10.5281/zenodo.23153

Maji, P.K. (2013). Neutrosophic soft set. Annals of Fuzzy Mathematics and Informatics, 5(1), 157-168.

Molodtsov, D. (1999). Soft set theory-first results. Computers \& Mathematics with Applications, 37(4-5), 19-31. https://doi.org/10.1016/S08981221(99)00056-5

Ozturk, T.Y., Aras, C.G. \& Bayramov, S. (2019). A new Approach to Operations on Neutrosophic Soft Sets and to Neutrosophic Soft Topological Spaces. Communications in Mathematics and Applications, 10(3), 481-493.

Smarandache, F. (2005). Neutrosophic set, a generalisation of the intuitionistic fuzzy sets. International Journal of Pure and Applied Mathematics, 24(3), 287-297. 